BP-PP-4-2

\title{
A study of biliary microbiota in patients with biliary tract disease
}

\author{
Min-Su PARK, Bumsoo KIM, Sangmok LEE
}

Department of Surgery, Kyunghee University, Seoul, Korea

Introduction: Biliary tract disease is associated with many factors: genes, environment, infection, etc. The current changes in biliary flora are thought to be involved in the formation of many gastrointestinal tract diseases. Therefore we want to investigate whether the biliary tract disease has a certain correlation with biliary microecology, and to detect specific strains.

Methods: Patients who were diagnosed with pancreaticobiliary cancer or benign inflammation were enrolled in this study. Mucosa from specimen or bile samples from endoscopic retrograde cholangiopancretography was utilized to collect for DNA extraction and 16S rRNA gene sequencing, followed by analysis of bile microbiota composition.

Results: A total of 52 adults were enrolled, of whom 8 with pancreaticobiliary cancer, 13 with acute inflammation, and 31 with chronic inflammation. We found that pancreaticobiliary cancer patients and bening inflammation patients shared similar stable and permanent dominant species and showed apparent differences in their biliary microbial composition and gene function. We discovered that Lactobacillaceae may potentially play a role in pancreaticobiliary cancer progression.

Conclusions: Our data suggested that changes in the microbiota between pancreaticobiliary cancer and benign inflammation may help deepen our understanding of the complex spectrum of different microbiotas involved in the development of pancreaticobiliary cancer. 